\title{
Genetic Spectrum and Variability in Chinese Patients with Amyotrophic Lateral Sclerosis
}

\author{
Zhi-Jun Liu",, Hui-Xia Lin 1,2,\#, Qiao Wei ${ }^{1, \#, ~ Q i-J i e ~ Z h a n g, " \#, ~ C o n g-X i n ~ C h e n ², ~ Q i n g-Q i n g ~ T a o ", ~}$ \\ Gong-Lu Liu', Wang Ni ${ }^{1}$, Aaron D. Gitler ${ }^{3}$, Hong-Fu Li ${ }^{1, *}$, Zhi-Ying Wu ${ }^{1, *}$ \\ ${ }^{1}$ Department of Neurology and Research Center of Neurology in Second Affiliated Hospital, and Key Laboratory \\ of Medical Neurobiology of Zhejiang Province, Zhejiang University School of Medicine, Hangzhou, China. \\ ${ }^{2}$ Department of Neurology and Institute of Neurology, First Affiliated Hospital, Fujian Medical University, \\ Fuzhou, China. \\ ${ }^{3}$ Department of Genetics, Stanford University School of Medicine, Stanford, CA, USA.
}

[Received January 2, 2019; Revised February 15, 2019; Accepted February 15, 2019]

\begin{abstract}
Amyotrophic lateral sclerosis (ALS) is a progressive, fatal neurodegenerative disease characterized by selective impairment of upper and lower motor neurons. We aimed to investigate the genetic spectrum and variability in Chinese patients with ALS. A total of 24 familial ALS (FALS) and 21 early-onset sporadic ALS (SALS) of Chinese ancestry were enrolled. Targeted next-generation sequencing (NGS) was performed in the probands, followed by verification by Sanger sequencing and co-segregation analysis. Clinical features of patients with pathogenic or likely pathogenic variants were present. The mutation frequency of ALS-related genes was then analyzed in Chinese population. In this cohort, 17 known mutations (9 SOD1, 5 FUS, 2 TARDBP and one SETX) were identified in 14 FALS and 6 early-onset SALS. Moreover, 7 novel variants (SOD1 c.112G $>$ C, OPTN c.811C > T, ERBB4 c.965T>A, DCTN1 c.1915C > T, NEFH c.2602G >A, NEK1 c.3622G >A, and TAF15 c.1535G>A) were identified. In southeastern Chinese FALS, the mutation frequency of SOD1, FUS, and TARDBP was 52.9\%, 8.8\%, $8.8 \%$ respectively. In early-onset SALS, FUS mutations were the most common (22.6\%). In Chinese ALS cases, p.H47R is most frequent $S O D 1$ mutations, while p.R521 is most common FUS mutation and p.M337V is most common TARDBP mutation. Our results revealed that mutations in SOD1, FUS and TARDBP are the most common cause of Chinese FALS, while FUS mutations are the most common cause of early-onset SALS. The genetic spectrum is different between Chinese ALS and Caucasian ALS.
\end{abstract}

Key words: amyotrophic lateral sclerosis, mutation, targeted next-generation sequencing, Chinese ancestry, genetic spectrum

Amyotrophic lateral sclerosis (ALS) is a progressive, fatal neurodegenerative disease characterized by selective impairment of upper and lower motor neurons, with or without cognitive impairment [1]. It predominantly occurs in adulthood with irreversible course. Patients usually suffer from progressive muscle weakness and atrophy and die of respiratory failure 3-5 years after the onset. No medications can halt the progression of ALS, although Riluzole prolongs survival by several months [2]. Approximately, 5-10\% of cases are familial ALS (FALS) with a Mendelian inheritance, while the remaining cases are sporadic ALS (SALS) [3]. The etiology of ALS has not been fully uncovered so far, but growing studies have revealed that genetic mutations played important roles in the pathogenesis of ALS, especially in juvenile-onset ALS [4]. In recent years,

*Correspondence should be addressed to: Dr. Zhi-Ying Wu (Email: zhiyingwu@zju.edu.cn) or Dr. Hong-Fu Li (Email: hongfuli@ zju.edu.cn), Department of Neurology, Second Affiliated Hospital, Zhejiang University School of Medicine, Hangzhou, China. \#These authors contributed equally to this study.

Copyright: ( $) 2019$ Liu ZJ et al. This is an open-access article distributed under the terms of the Creative Commons Attribution License, which permits unrestricted use, distribution, and reproduction in any medium, provided the original author and source are credited. 
evolving technologies for gene mapping and sequencing have facilitated the identification of multiple ALS genes. To date, more than 25 genes have been implicated in ALS [5,6]. Among these genes, C9ORF72, SOD1, FUS, and TARDBP are the most common causative genes [4]. Although mutations in some ALS-linked genes have been observed worldwide, geographic variability has also been observed. For example, the C9ORF72 hexanucleotide repeat expansion accounts for about $40 \%$ of FALS in North America and Europe [4]. while it is rare in the Chinese ALS population $[7,8]$. Such discrepancies might be attributed to differences in ethnicity and haplotype background.

In this study, we performed targeted next-generation sequencing (NGS) on a cohort of 24 FALS cases and 21 early-onset SALS cases from southeastern China. In addition, we integrated the previously reported ALS cases from our group and other Chinese groups to characterize the genetic spectrum of Chinese ALS patients. We summarize the mutation frequency and distribution of common ALS causative genes (SOD1, FUS, and $T A R D B P$ ) in Chinese population.

\section{MATERIALS AND METHODS}

\section{Participants}

A total of 24 FALS probands and 21 early-onset sporadic ALS were recruited from July 2008 to April 2017 in Second Affiliated Hospital, Zhejiang University School of Medicine, First Affiliated Hospital, Fujian Medical University, and Huashan Hospital, Fudan University. Majority of patients were from southeastern China. Age at onset under 40 years old was defined as early-onset in this study. Of the 24 FALS cases, five cases were previously reported, but no responsible mutation was found that time $[9,10]$. The diagnosis of ALS was made by at least two senior neurologists. All patients fulfilled the El Escorial criteria for ALS [11]. In view of the fact that a few patients with a diagnosis of suspected ALS had predominant lower motor neuron (LMN) symptoms, we excluded the possibility of spinal muscular atrophy (SMA) and spinal-bulbar muscular atrophy (SBMA) by screening $S M N 1$ and $A R$ gene in all patients. This study was approved by the ethics committee at each participating center. Written consents were obtained from all participants.

\section{Targeted sequencing}

Genomic DNA was extracted from EDTA-treated peripheral blood using DNA Extraction Kit (Qiagen, Hilden, Germany). Genetic analysis of the hexanucleotide repeats in C9ORF72 was completed as previously described [12]. A custom panel (Roche, Madison, USA) was designed to capture and sequence the 27 wellestablished ALS-related genes (Supplementary table 1). All the exons and flanking regions of each gene were covered by this panel. The targeted exomes of the prepared samples were captured by NimbleGen SeqCap EZ products (Roche, Madison, USA). The targeted libraries were indexed, polled and sequenced using Illumina Hiseq2500 system (Genergy Biotechnology Co. Ltd., Shanghai, China). Short reads were aligned to the human genome (UCSC hg19) using Burrows-Wheeler Aligner v0.7.15. The calling of variants, including single nucleotide variants (SNVs) and indels, were accomplished by Genome Analysis Toolkit v3.6. Sequencing depth and coverage analyses were performed using Genome Analysis Toolkit v3.6. The called variants were filtered by Hard filtering and Variant Quality Score Recalibration, and further annotated using ANNOVAR v2016Feb01.

Table 1. Demographic and clinical features of all ALS patients.

\begin{tabular}{lccc}
\hline & FALS & SALS & Total \\
\hline $\begin{array}{l}\text { Number of } \\
\text { subjects, } \mathrm{n}\end{array}$ & 24 & 21 & 45 \\
$\quad$ M/F, n & $13 / 11$ & $13 / 8$ & $26 / 19$ \\
$\quad$ M/F ratio & 1.2 & 1.6 & 1.4 \\
Age of onset, mean & & & \\
\pm SD (y) & & & \\
M & $34.5 \pm 13.1$ & $30.4 \pm 13.5$ & $32.4 \pm 13.2$ \\
F & $47.1 \pm 14.1$ & $31.3 \pm 8.1$ & $40.4 \pm 14.2$ \\
Total & $40.3 \pm 14.8$ & $30.7 \pm 11.5$ & $35.8 \pm 14.1$ \\
Site of onset, n & & & \\
$\quad$ Upper limbs & 9 & 11 & 20 \\
Lower limbs & 13 & 9 & 22 \\
Chest/abdomen & 1 & 0 & 1 \\
Bulbar & 1 & 1 & 2 \\
\hline
\end{tabular}

\section{Variant analysis}

All nonsynonymous variants covered more than 20x and within the coding and splicing regions were selected and screened with dbSNP, 1000 Genomes Project (1000G), NHLBI Exome Sequencing Project (ESP6500), and Exome Aggregation Consortium (ExAC). Potential functional consequences of the nonsynonymous variants were evaluated by SIFT, Mutationtaster, and PolyPhen-2, and CADD. The variants known to be pathogenic to the disease in any database of NCBI ClinVar, HGMD (Human Gene Mutation Database), and ALSoD (Amyotrophic Lateral Sclerosis Online Genetics Database) were picked out for further analysis. 
Table 2. The known pathogenic variants of ALS-related gene identified in this study.

\begin{tabular}{|c|c|c|c|}
\hline Gene & Nucleotide change & Amino acid change & Index cases (family history) \\
\hline SOD1 & c. $13 \mathrm{G}>\mathrm{T}$ & p.A5S & 1 (Yes) \\
\hline SOD1 & c. $14 \mathrm{C}>\mathrm{T}$ & p.A5V & 1 (Yes) \\
\hline SOD1 & c. $32 \mathrm{G}>\mathrm{T}$ & p.G11V & 1 (Yes) \\
\hline SOD1 & c. $49 \mathrm{G}>\mathrm{T}$ & p.G17C & 1 (Yes) \\
\hline SOD1 & c. $140 \mathrm{~A}>\mathrm{G}$ & p.H47R & 2 (Yes) \\
\hline SOD1 & c. $251 \mathrm{~A}>\mathrm{G}$ & p.D84G & 1 (Yes) \\
\hline SOD1 & c. $319 \mathrm{C}>\mathrm{G}$ & p.L107V & 1 (Yes) \\
\hline SOD1 & c. $363 \mathrm{~T}>\mathrm{G}$ & p.H121Q & 2 (Yes) \\
\hline SOD1 & c. $449 \mathrm{~T}>\mathrm{C}$ & p.I150T & 1 (Yes) \\
\hline FUS & c. $1483 C>T$ & p.R495X & 1 (Yes) \\
\hline FUS & c. $1528 \mathrm{~A}>\mathrm{G}$ & p.K510E & $1(\mathrm{No})$ \\
\hline FUS & c. $1561 \mathrm{C}>\mathrm{T}$ & p.R521C & $1(\mathrm{No})$ \\
\hline FUS & c. $1562 \mathrm{G}>\mathrm{A}$ & p.R521H & 1 (Yes) \\
\hline FUS & c. $1574 \mathrm{C}>\mathrm{T}$ & p.P525L & $2(\mathrm{No})$ \\
\hline$T A R D B P$ & c. $1009 A>G$ & p.M337V & 1 (Yes) \\
\hline$T A R D B P$ & c. $1069 \mathrm{G}>\mathrm{A}$ & p.G357S & $1(\mathrm{No})$ \\
\hline SETX & c. $1504 \mathrm{C}>\mathrm{T}$ & p.R502W & $1(\mathrm{No})$ \\
\hline
\end{tabular}

\section{Sanger sequencing}

Sanger sequencing was performed as previously described to verify the variants identified by targeted NGS [13]. Briefly, forward and reverse PCR primers were designed to amplify the fragments covering the variant sites. PCR products were purified with shrimp alkaline phosphatase and exonuclease and then directly sequenced on an ABI 3500xL Dx Genetic Analyzer (Applied Biosystems, Foster City, USA). Co-segregation analysis was performed in the families with identified mutations.

\section{RESULTS}

\section{Overview of demographics and clinical features}

A total of 24 FALS probands (13 males; 11 females) and 21 early-onset SALS (13 males; 8 females) were included in this study. The demographic and clinical features were summarized in Table 1. All patients are of Chinese Han origin and the majority of patients were from southeastern China. Among these patients, the mean age at onset was $35.8 \pm 14.1$ years (range from 7 to 65 ). Site of onset was upper limbs in 20 cases and lower limbs in 22 cases. Only two cases had bulbar onset and one case had abdomen onset.

\section{Targeted sequencing of ALS patient samples}

Among the 45 subjects recruited to our study, we subjected each DNA sample to next generation sequencing (NGS). An average of $93.25 \%$ target regions were sequenced with at least $30 \mathrm{x}$ coverage, and $86.18 \%$ target regions with 50x coverage. The mean coverage of the target regions per sample was 179.73. After filtering, a total of 29 different variants including 17 known pathogenic variants and 7 novel variants were identified (Table 2 and 3). All these variants were verified by Sanger sequencing.

\section{Seventeen known pathogenic variants were identified}

We assessed the C9ORF72 hexanucleotide repeat expansion by repeat-primed PCR, resulting in negative findings in all samples, consistent with the paucity of C9ORF72 mutation carriers in the Chinese ALS population [8]. A total of 17 known pathogenic variants, including 9 SODl mutations $(\mathrm{c} .13 \mathrm{G}>\mathrm{T}, \mathrm{c} .14 \mathrm{C}>\mathrm{T}$, c. $32 \mathrm{G}>\mathrm{T}$, с. $49 \mathrm{G}>\mathrm{T}$, c. $140 \mathrm{~A}>\mathrm{G}$, c. $251 \mathrm{~A}>\mathrm{G}$, c. $319 \mathrm{C}>\mathrm{G}$, c. $363 \mathrm{~T}>\mathrm{G}$ and c. $449 \mathrm{~T}>\mathrm{C}$ ), 5 FUS mutations (c. $1483 \mathrm{C}>\mathrm{T}$, c. $1528 \mathrm{~A}>\mathrm{G}$, c. $1561 \mathrm{C}>\mathrm{T}$, c. $1562 \mathrm{G}>\mathrm{A}$, and c. $1574 \mathrm{C}>\mathrm{T}), 2$ TARDBP mutations (c.1009A $>\mathrm{G}$ and c.1069G $>\mathrm{A}$ ), and one SETX mutation (c.1504C>T), were identified in 14 FALS cases and 6 early-onset sporadic cases (Table 2). In addition, we detected a previously reported SETX variant (c.4660T $>$ G, p.C1554G) in a sporadic case. This variant was documented in a few studies with controversial pathogenicity [14-16]. The frequency of this variant is 0.0058 in $1000 \mathrm{G}, 0.0032$ in ESP6500, and 0.0058 in ExAC. Co-segregation analysis revealed that this variant was also present in the patient's unaffected mother and younger brother. Therefore, this variant seems unlikely to be pathogenic.

\section{Seven novel variants were identified in ALS-related genes}

In addition, we identified 7 novel variants in 7 unrelated ALS cases, including 5 FALS and two early-onset SALS (Table 3). All these variants were absent or with low 
frequency in 1000G, ESP6500, and ExAC. Of these variants, one SOD1 variants (c. $112 \mathrm{G}>\mathrm{C})$ were identified in one FALS cases. The mutation was absent in 1000G, ESP6500, ExAC, and our 200 controls. In addition, it was predicted to be deleterious by SIFT, PolyPhen-2, Mutation Taster, and CADD. Then, another SOD1 mutation $(\mathrm{c} .112 \mathrm{G}>\mathrm{A})$ has been reported to be pathogenic (http://www.hgmd.cf.ac.uk/). According to ACMG standards, this variant was classified as pathogenic. Six variants $(O P T N$ c. $811 \mathrm{C}>\mathrm{T}, E R B B 4$ c. $965 \mathrm{~T}>\mathrm{A}, D C T N 1$ c. $1915 \mathrm{C}>\mathrm{T}, N E F H$ c. $2602 \mathrm{G}>\mathrm{A}, N E K 1$ c. $3622 \mathrm{G}>\mathrm{A}$, and TAF15 c. $1535 \mathrm{G}>\mathrm{A}$ ) of uncertain significance were identified in four FALS patients and two early-onset SALS patients. In addition, a novel variant (c. $-34 \mathrm{C}>\mathrm{A}$ ) in a noncoding region of $F U S$ was identified in a sporadic case.

Altogether, 17 known pathogenic variants and one novel pathogenic variant was detected in 15 FALS cases and 6 SALS cases. In addition, 6 variants of uncertain significance were detected in four FALS cases and two early-onset SALS cases. In total, 19 of 24 (79.2\%) FALS and 8 of $21(38.1 \%)$ early-onset SALS cases were identified to carry variants of ALS-related genes, while no variant was detected in the remaining cases.

Table 3. The novel variants of ALS-related genes identified in this study.

\begin{tabular}{|c|c|c|c|c|c|c|c|c|c|}
\hline Gene & Mutation & $1000 \mathrm{G}$ & ESP6500 & ExAC & SIFT & $\begin{array}{l}\text { Mutation } \\
\text { Taster }\end{array}$ & $\begin{array}{l}\text { Polyphen- } \\
2\end{array}$ & CADD & ACMG \\
\hline SOD1 & $\begin{array}{l}\text { c.112G>C } \\
\text { (p.G38R) }\end{array}$ & 0 & 0 & 0 & Damaging & $\begin{array}{l}\text { Disease } \\
\text { causing }\end{array}$ & $\begin{array}{l}\text { Probably } \\
\text { damaging }\end{array}$ & Damaging & $\mathrm{P}(\mathrm{PS} 1+\mathrm{PM} 1+\mathrm{PM} 2+\mathrm{PP} 2+\mathrm{PP} 3)$ \\
\hline OPTN & $\begin{array}{l}\text { c.811C>T } \\
\text { (p.R271C) }\end{array}$ & 0 & 0 & $1.65 \mathrm{E}-05$ & Tolerable & Polymorphism & Benign & Damaging & VUS (PM2) \\
\hline$E R B B 4$ & $\begin{array}{l}\text { c. } 965 \mathrm{~T}>\mathrm{A} \\
(\mathrm{p} . \mathrm{M} 322 \mathrm{~K})\end{array}$ & 0 & 0 & 0 & Tolerable & $\begin{array}{l}\text { Disease } \\
\text { causing }\end{array}$ & Benign & Tolerable & VUS (PM1+PM2+PP2) \\
\hline DCTN1 & $\begin{array}{l}\text { c. } 1915 \mathrm{C}>\mathrm{T} \\
(\mathrm{p} . \mathrm{R} 639 \mathrm{~W})\end{array}$ & 0 & 0 & 4.99E-05 & Damaging & $\begin{array}{l}\text { Disease } \\
\text { causing }\end{array}$ & $\begin{array}{l}\text { Probably } \\
\text { damaging }\end{array}$ & Damaging & VUS (PM1+PM2+PP3) \\
\hline$N E F H$ & $\begin{array}{l}\text { c. } 2602 \mathrm{G}>\mathrm{A} \\
\text { (p.E868K) }\end{array}$ & 0 & 0 & 0 & Tolerable & $\begin{array}{l}\text { Disease } \\
\text { causing }\end{array}$ & $\begin{array}{l}\text { Probably } \\
\text { damaging }\end{array}$ & Tolerable & VUS (PM2+PP3+BP1) \\
\hline NEK1 & $\begin{array}{l}\text { c. } 3622 \mathrm{G}>\mathrm{A} \\
\text { (p.D1208N) }\end{array}$ & 0 & 0 & $1.06 \mathrm{E}-04$ & Damaging & $\begin{array}{l}\text { Disease } \\
\text { causing }\end{array}$ & $\begin{array}{l}\text { Probably } \\
\text { damaging }\end{array}$ & Damaging & VUS (PM2) \\
\hline$T A F 15$ & $\begin{array}{l}\text { c. } 1535 \mathrm{G}>\mathrm{A} \\
\text { (p.R512Q) }\end{array}$ & 0 & 7.7E-05 & $9.15 \mathrm{E}-05$ & Damaging & $\begin{array}{l}\text { Disease } \\
\text { causing }\end{array}$ & $\begin{array}{l}\text { Probably } \\
\text { damaging }\end{array}$ & Damaging & VUS \\
\hline
\end{tabular}

Abbreviations: 1000G=1000 Genomes Project; ESP6500= NHLBI exome sequencing project; ExAC= Exome Aggregation Consortium.

\section{Clinical features of the patients carrying pathogenic variants}

The clinical features of the patients carrying pathogenic variants or likely pathogenic variants are summarized in Table 4. The average age at onset is $37.81 \pm 15.20$ years, ranging from 13 to 65 years. Among the 21 patients carrying mutations, 19 cases presented with limb-onset symptoms, one case exhibited bulbar onset, and the other one initially developed weakness of abdominal muscles. Only one patient carrying a $F U S$ p.K510E mutation exhibited cognitive decline four months after disease onset.

In the current study, 50\% (12/24) of FALS cases carried SOD1 mutations, including 9 known pathogenic variants (p.A5S, p.A5V, p.G11V, p.G17C, p.H47R, p.N84G, p.L107V, p.H121Q and p.I150T) and one novel pathogenic variant (c.112G $>$ C, p.G38R). The average age of onset of SODI mutation carriers was $42.75 \pm 13.44$ years. The site of onset in SOD1 mutation carriers was in the lower limbs $(75 \%, n=9)$, upper limbs $(16.7 \%, n=2)$, and abdominal muscle $(8.3 \%, \mathrm{n}=1)$. These patients typically presented with dominant lower motor neuron signs. Interestingly, slow progression was significantly observed in the patients carrying SOD1 p.G38R, p.H47R, or p.D84G mutations.

Additionally, we found 5 known FUS mutations (p.R495X, p.K510E, p.R521C, p.R521H, and p.P525L) in two FALS cases and four SALS cases. The average age at onset in patients with FUS mutations was $25.67 \pm 7.61$ years; significantly lower than that in patients with SOD1 mutations. The patients carrying p.R521C or p.R521H mutation manifested predominant lower motor neuron signs. Interestingly, neck and proximal muscle weakness which usually occurs in p.R521C mutation carriers was also observed in our patient with p.R521H mutation. In line with previous reports [17-19], the patient with the p.R495X mutation showed an early-onset and rapid disease progression.

Moreover, we detected two known TARDBP mutations, p.M337V and p.G357S. The patient with p.M337V mutation exhibited onset of bulbar symptoms, while the patient with p.G357S had spine onset. Interestingly, we identified a known SETX variant (p.R502W) in a SALS patient, who predominantly presented muscle weakness and atrophy of proximal lower limbs and exhibited a slowly progressive disease course without any evidence of upper motor neuron signs. 
Table 4. The clinical features of the patients carrying pathogenic variants or likely pathogenic variants.

\begin{tabular}{|c|c|c|c|c|c|c|c|c|}
\hline Patients & Mutation & $\begin{array}{l}\text { Family } \\
\text { history }\end{array}$ & Gender & $\begin{array}{l}\text { Age at } \\
\text { onset }(y)\end{array}$ & $\begin{array}{l}\text { Site of } \\
\text { onset }\end{array}$ & $\begin{array}{l}\text { Disease } \\
\text { duration (m) }\end{array}$ & $\begin{array}{l}\text { Age of } \\
\text { death }(y)\end{array}$ & Predominant features \\
\hline 1 & SOD1: c.13G>T & Yes & $\mathrm{M}$ & 29 & LL & $>4$ & alive & LMN dominance \\
\hline 2 & SOD1: c. $14 \mathrm{C}>\mathrm{T}$ & Yes & $\mathrm{F}$ & 60 & UL & 15 & 61 & Classical ALS \\
\hline 3 & SOD1: c.32G>T & Yes & $\mathrm{F}$ & 25 & LL & $>10$ & alive & Classical ALS \\
\hline 4 & SOD1: c. $49 \mathrm{G}>\mathrm{T}$ & Yes & M & 49 & Abdomen & 38 & 52 & LMN dominance \\
\hline 5 & SOD1: c. $112 \mathrm{G}>\mathrm{C}$ & Yes & M & 26 & LL & $>120$ & alive & $\begin{array}{l}\text { LMN dominance; slow } \\
\text { progression }\end{array}$ \\
\hline 6 & SOD1: c. $140 \mathrm{~A}>\mathrm{G}$ & Yes & $\mathrm{F}$ & 60 & UL & $>180$ & alive & $\begin{array}{l}\text { LMN; very slow } \\
\text { progression }\end{array}$ \\
\hline 7 & SOD1: c. $140 \mathrm{~A}>\mathrm{G}$ & Yes & M & 53 & LL & $>21$ & alive & LMN dominance \\
\hline 8 & SOD1: c. $251 \mathrm{~A}>\mathrm{G}$ & Yes & M & 32 & LL & $>29$ & alive & $\begin{array}{l}\text { LMN dominance; slow } \\
\text { progression }\end{array}$ \\
\hline 9 & SOD1: c. $319 \mathrm{C}>\mathrm{G}$ & Yes & M & 41 & LL & $>8$ & alive & Classical ALS \\
\hline 10 & SOD1: c.363T>G & Yes & $\mathrm{F}$ & 42 & LL & 51 & 46 & LMN dominance \\
\hline 11 & SOD1: c.363T $>\mathrm{G}$ & Yes & $\mathrm{F}$ & 60 & LL & 27 & 62 & Classical ALS \\
\hline 12 & SOD1: c.449T>C & Yes & $\mathrm{F}$ & 36 & LL & 12 & 37 & Classical ALS \\
\hline 13 & FUS: c. $1483 \mathrm{C}>\mathrm{T}$ & Yes & M & 25 & LL & 9 & 26 & Fast progression \\
\hline 14 & FUS: c. $1528 \mathrm{~A}>\mathrm{G}$ & No & M & 26 & UL & $>14$ & alive & $\begin{array}{l}\text { LMN dominance; } \\
\text { cognitive decline }\end{array}$ \\
\hline 15 & FUS: c. $1561 \mathrm{C}>\mathrm{T}$ & No & M & 30 & LL & 41 & 33 & LMN dominance \\
\hline 16 & FUS: c. $1562 \mathrm{G}>\mathrm{A}$ & Yes & $\mathrm{F}$ & 36 & UL & $>16$ & alive & $\begin{array}{l}\text { Classical ALS; dropped } \\
\text { head syndrome }\end{array}$ \\
\hline 17 & FUS: c. $1574 \mathrm{C}>\mathrm{T}$ & No & $\mathrm{F}$ & 13 & LL & $>40$ & alive & Classical ALS \\
\hline 18 & FUS: c. $1574 \mathrm{C}>\mathrm{T}$ & No & M & 24 & UL & $>27$ & alive & Classical ALS \\
\hline 19 & $\begin{array}{l}\text { TARDBP: } \\
\text { c. } 1009 \mathrm{~A}>\mathrm{G}\end{array}$ & Yes & M & 44 & Bulbar & $>156$ & alive & $\begin{array}{l}\text { LMN dominance; slow } \\
\text { progression }\end{array}$ \\
\hline 20 & $\begin{array}{l}\text { TARDBP: } \\
\text { c. } 1069 \mathrm{G}>\mathrm{A}\end{array}$ & No & M & 65 & UL & $>24$ & alive & Classical ALS \\
\hline 21 & SETX: c. $1504 \mathrm{C}>\mathrm{T}$ & No & M & 18 & LL & $>84$ & alive & $\begin{array}{l}\text { LMN dominance; slow } \\
\text { progression }\end{array}$ \\
\hline
\end{tabular}

Abbreviations: $\mathrm{M}=$ male; $\mathrm{F}=$ female; $\mathrm{LL}=$ lower limb; UL=upper limb; $\mathrm{LMN}=$ lower motor neuron; $\mathrm{ALS}=$ amyotrophic lateral sclerosis.

\section{Genetic spectrum in our ALS patients from southeastern China}

We previously reported 10 unrelated FALS patients with mutations identified in SOD1 (4 mutations/6 patients), TARDBP (2/2), DCTN1 (1/1), and FUS (1/1) [9,10,20,21]. As shown in Figure 1A, when integrating these results into the current study, we found $85.3 \%$ (29/34) of FALS carried mutation. SOD1 mutations were the most common $(18 / 34,52.9 \%)$ in our FALS cases, followed by FUS $(3 / 34,8.8 \%)$ and TARDBP $(3 / 34,8.8 \%)$ mutations. Despite being an uncommon cause, DCTN1 mutations were identified in $2.9 \%(1 / 34)$ of FALS patients. However, $11.8 \%$ (4/34) of FALS cases carried a variant of unknown significance and $14.7 \%$ (5/34) of FALS patients were not identified potential pathogenic variants. In addition, we identified three FUS mutations in four SALS cases and one TARDBP mutation in one SALS case. Integrating our previously reported three $F U S$ mutations in 3 of 10 juvenile-onset SALS cases [20], we found the frequency for FUS mutation and TARDBP mutation in early-onset SALS were $22.6 \%$ (7/31) and $3.2 \%(1 / 31)$, respectively. Also, one SETX mutation was detected in one SALS patient and two variants of uncertain significance were detected in two SALS cases. In total, $35.5 \%$ (11/31) of early-onset SALS patients were identified to carry potential pathogenic variants whereas the remaining $64.5 \%(20 / 31)$ of SALS patients were not.

\section{Mutation frequency of common ALS genes in Chinese population}

To analyze mutation frequency and distribution of common ALS causative genes (SODI, FUS, and $T A R D B P$ ) in Chinese population, we reviewed the published genetic reports on Chinese ALS patients. In total, 36 additional studies reporting mutations of SOD1, $F U S$, and TARDBP in Chinese ALS patients were analyzed. As shown in Supplementary table 2, a total of 73 different SOD1 mutations were reported in Chinese ALS patients (Fig. 1B). Among these mutations, p.H47R was detected in 14 unrelated patients, representing the most frequent $S O D 1$ mutation in Chinese ALS patients. In addition, SOD1 mutations p.G42D, p.L107F, and p.C112Y are prevalent. The most common SODI mutations in North America (p.A5V) and worldwide 
(p.D91A) were rare in Chinese ALS patients. A total of 15 FUS mutations including 6 indels, 8 missense mutations and one nonsense mutation were reported in Chinese ALS patients (Fig. 1C). The p.R521 mutation (including p.R521G, p.R521H, p.R521L and p.R521C) was found in 9 unrelated ALS patients, representing the most frequent FUS mutation in Chinese ALS patients. In addition, 10 TARDBP missense mutations were reported in Chinese ALS patients (Fig. 1D), all of which were located in exon 6. Of these TARDBP mutations, p.M337V seems to be a high frequency mutation in Chinese ALS patients. In early-onset SALS, 5 cases with SOD1 mutations, 16 cases with FUS mutations, and one case with TARDBP mutation had been described.
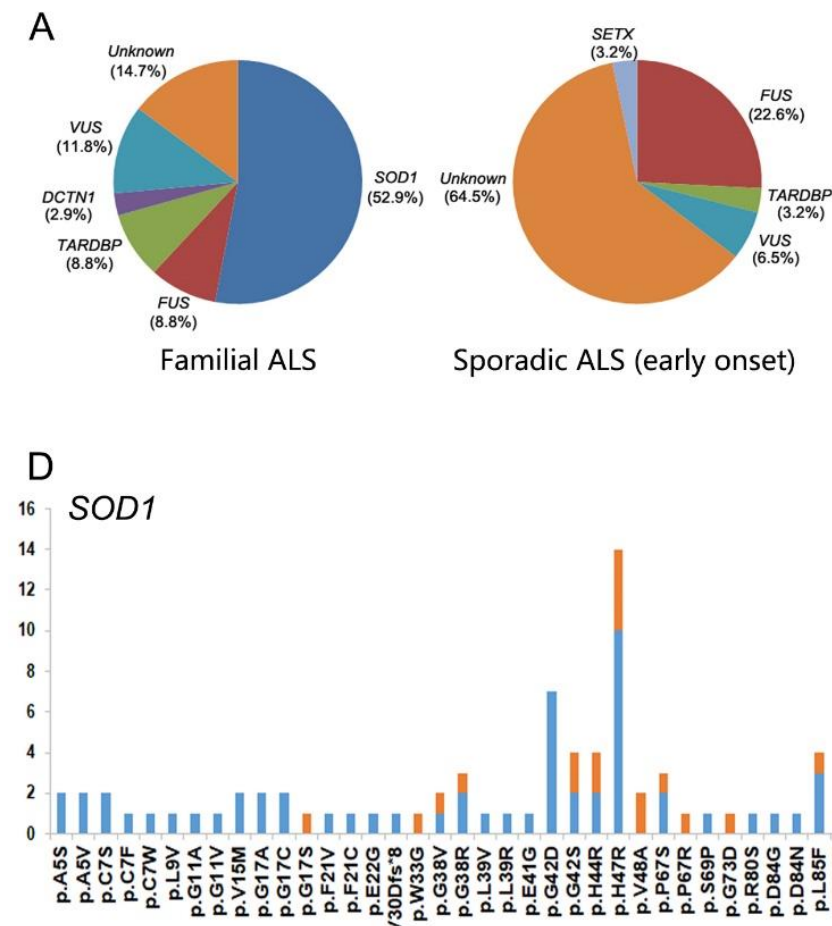
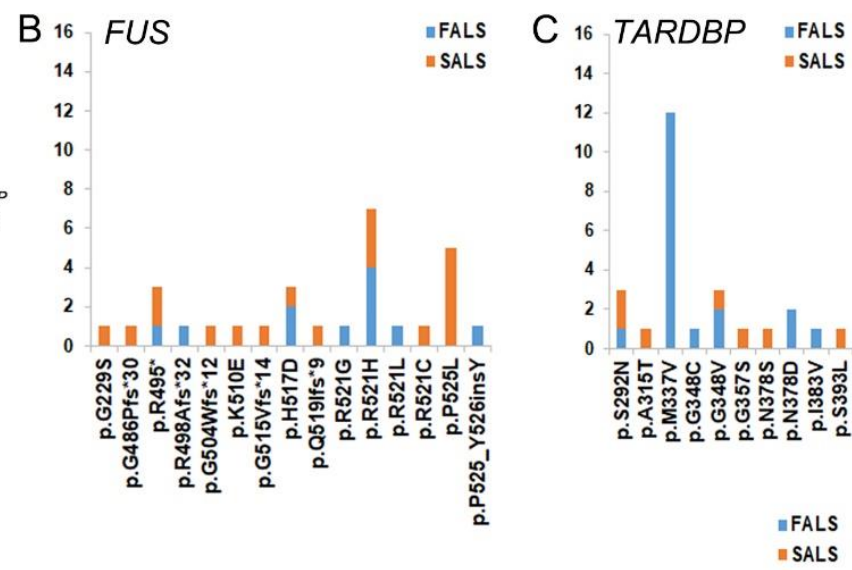

Figure 1. Mutation spectrum of ALS-linked genes in our ALS cohort and Chinese ALS patients. (A) In our ALS cohort, the mutation frequency of SOD1, FUS, TARDBP, and DCTN1 in FALS was $52.9 \%, 8.8 \%, 8.8 \%$, and 2.9\%, respectively. FUS mutations were the most common cause of early-onset SALS (25.8\%), while $61.3 \%$ of the cases were not identified pathogenic mutations. (B) The mutation frequency of $F U S$ in Chinese ALS cases. (C) The mutation frequency of TARDBP in Chinese ALS cases. (D) The mutation frequency of SOD1 in Chinese ALS cases.

\section{DISCUSSION}

In this study, we performed targeted NGS and Sanger sequencing in a Chinese cohort of 24 FALS cases and 21 early-onset SALS cases. In total, we identified 17 known pathogenic variants and a novel SOD1 pathogenic variants $(\mathrm{c} .112 \mathrm{G}>\mathrm{C})$ in 15 FALS cases and 6 early-onset SALS cases. In addition, 6 variants of uncertain significance were identified in four FALS cases and two SALS cases. The pathogenicity of these 6 novel variants remains to be determined. When integrating our previously reported ALS cases with mutation
[9,10,20,21], we found that $85.3 \%$ (29/34) of FALS carried mutations of ALS-linked genes. However, we failed to detect pathogenic mutations in the majority $(64.5 \%, 20 / 31)$ of early-onset SALS cases. Among these mutation carriers, SODI mutation was the most common cause of FALS, which is consistent with previous studies $[22,23,24,25]$. FUS mutation accounted for the most frequent in the early-onset SALS cases. Previous studies of Chinese SALS which included patients with a wide range of age of onset indicated that FUS mutations are also the most frequent cause of SALS, but the frequency of FUS mutation in SALS is lower than that in early-onset 
SALS $(25.8 \%)$, ranging from $1.7 \%$ to $1.9 \%[23,24]$. However, none FUS mutations was identified in the cohorts of SALS from central-southern and northern China $[25,26]$. Of note, no $C 9 O R F 72$ repeat expansion were identified in our cohort, further supporting the finding that C9ORF72 mutation is rare in the Chinese population [8].

As the first causative gene for ALS, SOD1 mutations are very common in both FALS (20\%) and SALS (1 2\%) [27]. To date, more than 185 SOD1 mutations have been described worldwide and p.D91A (previously described as p.D90A) is the most common one [28]. However, this mutation was reported in only one Chinese ALS case [23]. Another mutation p.A5V (previously described as p.A4V), which accounts for up to $50 \%$ of $S O D 1$ mutations in North America, is rarely described in Chinese ALS cases [29]. Moreover, it is reported that the p.A5V mutation carrier did not share the common founder effect observed in North America [29]. In contrast, p.H47R was the most common SOD1 mutation in Chinese ALS cases, which is similar to that in Japan [30]. In addition, p.G42D, p.L107F, and p.C112Y, were more frequent than other mutations. These results imply that the mutation spectrum of SOD1 was different between Chinese ALS and Caucasian ALS. In regard to the clinical features, cognitive impairment and bulbar onset was not common in Chinese patients with SOD1 mutations, which is consistent with previous reports [30]. However, phenotypic heterogeneity was observed not only in cases with different SODI mutations, but also in familial members with the same SODI mutation.

Compared to the high frequency of SODI mutations in Chinese FALS cases, FUS mutations were not common. However, FUS remains the most frequent genetic determinant of early-onset SALS. Previous study in China demonstrated that mutations in the FUS are the most frequent genetic cause in juvenile SALS (age of onset $<25$ years) of Chinese origin [31]. This is consistent with our results. The frequency of FUS mutations in earlyonset SALS is similar between Chinese and Caucasian population [32]. Of note, over half of FUS mutations in Chinese cases were frameshift mutations. Age at onset is young in most of the cases, and most of FUS mutation carriers manifested predominant lower motor neuron symptoms and a rapid disease progression. In addition, 9 mutations located in exon 6 of TARDBP were reported in ALS patients of Chinese ancestry and p.M337V seems to be the most common one, implying TARDBP is one of the major causative genes in Chinese ALS cases.

In summary, we investigated the genetic mutations in a Chinese cohort with ALS and characterize the genetic spectrum of Chinese ALS. Our results revealed that SOD1 mutations are the most common cause of Chinese FALS, followed by TARDBP, FUS and DCTN1. FUS mutations remained the most frequent genetic determinant of Chinese early-onset SALS. In the future, it will be interesting to explore the basis for variability in mutation frequencies between Chinese ALS and Caucasian ALS.

\section{Acknowledgments}

The authors sincerely thank the participants for their help and willingness to participate in this study. This work was supported by the grants $(81671245,81125009)$ to ZhiYing $\mathrm{Wu}$ from the National Natural Science Foundation, and the research foundation for distinguished scholar of Zhejiang University to Zhi-Ying Wu (188020193810101/089, Hangzhou).

\section{Conflicts of interest}

The authors report no potential conflicts of interest.

\section{Supplementary Materials}

The Supplemenantry data can be found online at: www.aginganddisease.org/EN/10.14336/AD.2019.0215.

\section{References}

[1] van Es MA, Hardiman O, Chio A, et al. (2017). Amyotrophic lateral sclerosis. Lancet, 390: 2084-2098.

[2] Bensimon G, Lacomblez L, Meininger V (1994). A controlled trial of riluzole in amyotrophic lateral sclerosis. ALS/Riluzole Study Group. N Engl J Med, 330: 585-591.

[3] Forbes RB, Colville S, Swingler RJ, Scottish ALSMNDR (2004). The epidemiology of amyotrophic lateral sclerosis (ALS/MND) in people aged 80 or over. Age Ageing, 33: 131-134.

[4] Renton AE, Chio A, Traynor BJ (2014). State of play in amyotrophic lateral sclerosis genetics. Nat Neurosci, 17: 17-23.

[5] Peters OM, Ghasemi M, Brown RH, Jr (2015). Emerging mechanisms of molecular pathology in ALS. J Clin Invest, 125: 2548.

[6] Therrien M, Dion PA, Rouleau GA (2016). ALS: Recent Developments from Genetics Studies. Curr Neurol Neurosci Rep, 16: 59.

[7] Chen L, Zhang B, Chen R, et al. (2015). Natural history and clinical features of sporadic amyotrophic lateral sclerosis in China. J Neurol Neurosurg Psychiatry, 86: 1075-1081.

[8] He J, Tang L, Benyamin B, et al. (2015). C9orf72 hexanucleotide repeat expansions in Chinese sporadic amyotrophic lateral sclerosis. Neurobiol Aging, 36: 2660 e2661-2668.

[9] Liu ZJ, Li HF, Tan GH, et al. (2014). Identify mutation in amyotrophic lateral sclerosis cases using HaloPlex target enrichment system. Neurobiol Aging, 35: 2881 e2811-2881 e2815. 
[10] Xiong HL, Wang JY, Sun YM, et al. (2010). Association between novel TARDBP mutations and Chinese patients with amyotrophic lateral sclerosis. BMC Med Genet, 11: 8.

[11] Brooks BR, Miller RG, Swash M, Munsat TL, World Federation of Neurology Research Group on Motor Neuron D (2000). El Escorial revisited: revised criteria for the diagnosis of amyotrophic lateral sclerosis. Amyotroph Lateral Scler Other Motor Neuron Disord, 1: 293-299.

[12] DeJesus-Hernandez M, Mackenzie IR, Boeve BF, et al. (2011). Expanded GGGGCC hexanucleotide repeat in noncoding region of C9ORF72 causes chromosome 9plinked FTD and ALS. Neuron, 72: 245-256.

[13] Dong Y, Ni W, Chen WJ, et al. (2016). Spectrum and Classification of ATP7B Variants in a Large Cohort of Chinese Patients with Wilson's Disease Guides Genetic Diagnosis. Theranostics, 6: 638-649.

[14] Arning L, Epplen JT, Rahikkala E, Hendrich C, Ludolph AC, Sperfeld AD (2013). The SETX missense variation spectrum as evaluated in patients with ALS4-like motor neuron diseases. Neurogenetics, 14: 53-61.

[15] Hirano M, Quinzii CM, Mitsumoto H, et al. (2011). Senataxin mutations and amyotrophic lateral sclerosis. Amyotroph Lateral Scler, 12: 223-227.

[16] Nishiyama A, Niihori T, Warita H, et al. (2017). Comprehensive targeted next-generation sequencing in Japanese familial amyotrophic lateral sclerosis. Neurobiol Aging, 53: 194 e191-194 e198.

[17] Waibel S, Neumann M, Rabe M, Meyer T, Ludolph AC (2010). Novel missense and truncating mutations in FUS/TLS in familial ALS. Neurology, 75: 815-817.

[18] Waibel S, Neumann M, Rosenbohm A, et al. (2013). Truncating mutations in FUS/TLS give rise to a more aggressive ALS-phenotype than missense mutations: a clinico-genetic study in Germany. Eur J Neurol, 20: 540546.

[19] Yan J, Deng HX, Siddique N, et al. (2010). Frameshift and novel mutations in FUS in familial amyotrophic lateral sclerosis and ALS/dementia. Neurology, 75: 807814.

[20] Liu ZJ, Lin HX, Liu GL, et al. (2017). The investigation of genetic and clinical features in Chinese patients with juvenile amyotrophic lateral sclerosis. Clin Genet, 92: 267-273.
[21] Niu YF, Xiong HL, Wu JJ, Chen Y, Qiao K, Wu ZY (2011). [Screening of mutations in SOD1 gene and analysis of genotype-phenotype correlation in Chinese patients with amyotrophic lateral sclerosis]. Yi Chuan, 33: 720-724.

[22] Tsai CP, Soong BW, Lin KP, Tu PH, Lin JL, Lee YC (2011). FUS, TARDBP, and SOD1 mutations in a Taiwanese cohort with familial ALS. Neurobiol Aging, 32:553 e13-e21.

[23] Zou ZY, Liu MS, Li XG, Cui LY (2016). The distinctive genetic architecture of ALS in mainland China. J Neurol Neurosurg Psychiatry, 87: 906-907.

[24] Liu Q, Liu F, Cui B, et al (2016). Mutation spectrum of Chinese patients with familial and sporadic amyotrophic lateral sclerosis. J Neurol Neurosurg Psychiatry, 87:1272.

[25] Hou L, Jiao B, Xiao T, et al (2016). Screening of SOD1, FUS and TARDBP genes in patients with amyotrophic lateral sclerosis in central-southern China. Sci Rep, 6:32478

[26] Zhang H, Cai W, Chen S, et al (2018). Screening for possible oligogenic pathogenesis in Chinese sporadic ALS patients. Amyotroph Lateral Scler Frontotemporal Degener, 19:419-425.

[27] Baumer D, Talbot K, Turner MR (2014). Advances in motor neurone disease. J R Soc Med, 107: 14-21.

[28] Li HF, Wu ZY (2016). Genotype-phenotype correlations of amyotrophic lateral sclerosis. Transl Neurodegener, 5: 3.

[29] Tang L, Ma Y, Liu X, Chen L, Fan D (2018). Identification of an A4V SOD1 mutation in a Chinese patient with amyotrophic lateral sclerosis without the A4V founder effect common in North America. Amyotroph Lateral Scler Frontotemporal Degener, 19: 466-468.

[30] Yamashita S, Ando Y (2015). Genotype-phenotype relationship in hereditary amyotrophic lateral sclerosis. Transl Neurodegener, 4: 13.

[31] Zou ZY, Liu MS, Li XG, Cui LY (2016). Mutations in FUS are the most frequent genetic cause in juvenile sporadic ALS patients of Chinese origin. Amyotroph Lateral Scler Frontotemporal Degener, 17: 249-252.

[32] Hubers A, Just W, Rosenbohm A, et al. (2015). De novo FUS mutations are the most frequent genetic cause in early-onset German ALS patients. Neurobiol Aging, 36: 3117 e3111-3117 e3116. 